\title{
Hashimoto's thyroiditis Autoimmune Disease: background and current status, update overview of Biotechnological and Biomedical fields and future trends for 3D models
}

\author{
Arnaud Martino Capuzzo \\ Department of Veterinary Medicine, University of Milan, Italy
}

\begin{abstract}
Hashimoto thyroiditis, also known as chronic autoimmune thyroiditis or chronic lymphocytic thyroiditis, is an autoimmune illness in which thyroid cells are damaged by immunological mechanisms involving cells and antibodies. Thyroid peroxidase and/or thyroglobulin autoantibodies in the serum are biochemical indicators of the condition, with females having a higher incidence than males and increasing with age. It's the leading cause of hypothyroidism in affluent countries. Inadequate dietary iodine intake, on the other hand, is the most common cause of hypothyroidism worldwide. The development of antithyroid antibodies that target the thyroid tissue, causing gradual fibrosis, is the pathogenesis of Hashimoto thyroiditis. The diagnosis can be difficult, and as a result, the problem is frequently not detected until late in the disease process. The most prevalent laboratory findings are raised TSH and low thyroxine (T4) levels, as well as enhanced antithyroid peroxidase (anti-TPO) antibodies. The pathogenesis, diagnosis, and management of Hashimoto thyroiditis are discussed in this article.
\end{abstract}

Keywords: Thyroid, Organoid, Spheroid, Biotechnology, Biomedical.

\section{Introduction}

Autoimmune thyroid diseases (AITDs) are the most frequent autoimmune diseases, affecting roughly $5 \%$ of the general population and predominating in women. AITDs are the most common cause of acquired thyroid disease during infancy and adolescence, despite reaching a peak in maturity. AITDs include Hashimoto's thyroiditis (HT) and Graves' disease (GD), both of which are characterized by thyroid infiltration by $\mathrm{T}$ and $\mathrm{B}$ cells that respond against thyroid antigens and the production of thyroid autoantibodies (Malkowski et al., 2019). Hashimoto thyroiditis is an 
autoimmune illness that damages thyroid cells through immunological mechanisms mediated by cells and antibodies. It's the leading cause of hypothyroidism in affluent countries. In contrast, the most prevalent cause of hypothyroidism worldwide is a lack of iodine in the diet. This condition is also known as chronic lymphocytic thyroiditis and chronic autoimmune thyroiditis (Aurizio et al., 2015). The pathogenesis of the disease is characterized by the production of antithyroid antibodies, which target thyroid tissue and cause progressive fibrosis. Diagnosis is frequently difficult and may not occur until late in the disease phase. The most prevalent laboratory results include elevated thyroid-stimulating hormone (TSH) levels and low levels of free thyroxine (fT4), as well as enhanced antithyroid peroxidase (TPO) antibodies (Brown et al., 2012). Patients may show signs, symptoms, and laboratory findings of hyperthyroidism or normal levels earlier in the course of the disease. This is due to the fact that thyroid gland cell death can be intermittent. Women are disproportionately affected. Women outnumber men by a factor of at least ten. Although some sources suggest that diagnosis occurs more frequently in the fifth decade of life, the majority of women are diagnosed between the ages of 30 and 50 (Brown et al., 2012). Levothyroxine, at a recommended dose of 1.6 to $1.8 \mathrm{mcg} / \mathrm{kg} / \mathrm{day}$, is the standard treatment. In the human body, T4 is converted to T3, which is the active form of thyroid hormone. Excessive supplementing can have negative and morbid consequences, including arrhythmias (the most prevalent of which is atrial fibrillation) and osteoporosis. The pathogenesis, diagnosis, and management of Hashimoto thyroiditis are discussed in this article (Aurizio et al., 2015).

\section{Background of Hashimoto's thyroiditis}

Hashimoto's thyroiditis is named after Japanese physician Hakaru Hashimoto of Kyushu University's medical school, who was the first to describe the symptoms of people with Struma lymphomatosa, a severe lymphocyte infiltration of the thyroid gland. In addition, all of the data in his first study came from four women. These findings explained the clinical features seen in these women, including lymphoid and plasma cell infiltration, lymphoid follicle development with germinal centers, fibrosis, degraded thyroid epithelial cells, and leukocytes in the lumen (Pastuszak et al., 2012). Despite Dr. Hashimoto's discovery and publishing, the disease was not recognized as separate from Reidel's Thyroiditis, a widespread disease in Europe at the time. Despite the fact that many earlier investigations had been documented and published, Hashimoto's struma lymphomatosa was only recognized as an early phase of Reidel's Thyroiditis in the early 1900s. It 
wasn't until 1931 that the disease was recognized as a separate entity, when Cleveland researchers Allen Graham characterized the symptoms and presentation in the same way as Hakuru did (Iddah and Macharia, 2013). Drs. Rose and Witebsky were able to show in 1956 that immunizing specific mice with thyroid extracts from other rodents mimicked the condition Hakuru and other researchers were seeking to describe. Anti-thyroglobulin antibodies were also identified in blood serum samples from the same animals by these specialists (Cappa et al., 2011). Researchers from the Middlesex Hospital in London were able to conduct human studies on individuals with comparable symptoms later that year. They were able to determine that these unwell patients have an immunological reaction to human thyroglobulin after purifying anti-thyroglobulin antibodies from their serum. Based on these information, it was hypothesized that Hashimoto's struma is an autoimmune thyroid disease (Brown et al., 2012).

\section{Risk factors}

These factors may increase your chances of getting Hashimoto's disease:

- Sex. Hashimoto's disease is significantly more common in women.

- Age. Hashimoto's disease can strike at any age, but it is most frequent in middle life.

- Heredity. If you have a family history of thyroid or other autoimmune diseases, you're more likely to get Hashimoto's disease.

- Other autoimmune disease. You're more likely to acquire Hashimoto's disease if you have another autoimmune condition like rheumatoid arthritis, type 1 diabetes, or lupus.

- Radiation exposure. Hashimoto's disease is more common in people who are exposed to high levels of environmental radiation.

\section{Hashimoto's stage}

Hashimoto's thyroiditis is a type of autoimmune disease that affects the thyroid gland. This means that our immune system sees the thyroid gland as a foreign intruder and begins to assault it, resulting in thyroid tissue loss. The person's ability to create thyroid hormone will be lost if this degradation continues for a long time. Hashimoto's disease is rarely diagnosed until it has progressed to the advanced stages, when the thyroid gland has been severely damaged. Wiersinga and colleagues identified five stages of Hashimoto's disease in 2014 (Yamada et al., 2014). 


\section{Thyroid Gland Anatomy and Histology}

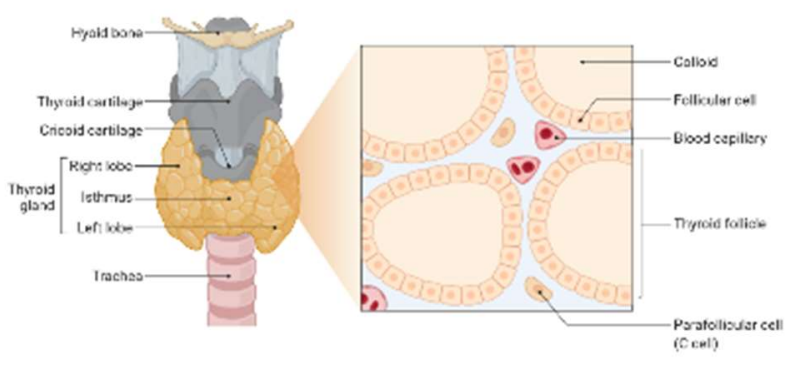

\section{Stages of Hashimoto's thyroiditis}

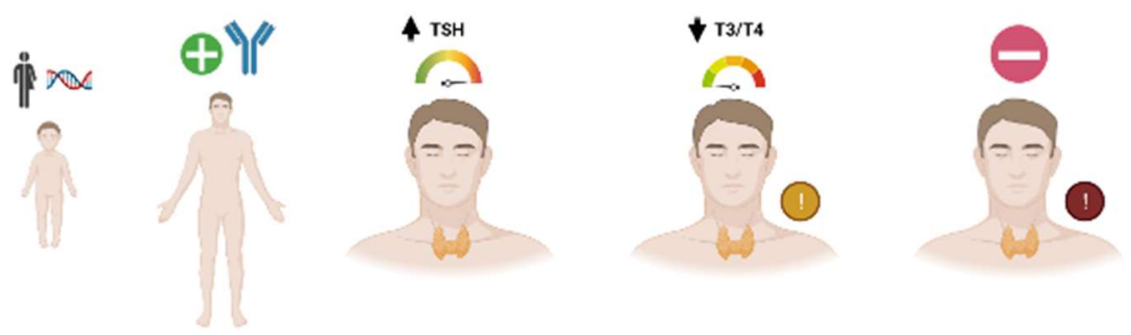

Fig. 1 Thyroid Gland Anatomy and Histology and 5 Stages of Hashimoto autoimmune thyroid disease:1) A person has a genetic predisposition to develop Hashimoto's and thyroid function is normal, without attack on the thyroid. 2) A person may test positive for thyroid antibodies and may have changes consistent with Hashimoto's but have normal TSH level. 3) Thyroid glands start to lose its ability to make enough hormone and there will be a slightly elevated TSH with normal T3/T4. More symptoms will be seen at this stage. 4) Thyroid glands have fully lost its

ability to compensate, elevate TSH and low T3/T4 levels. With overt hypothyroidism, Hashimoto's are often diagnosed. 5) Fully progression of the autoimmune response and other types of autoimmune conditions may develop. Created with BioRender.com

\section{Clinical criteria}

Doctors may test for Hashimoto's disease if you're feeling increasingly tired or sluggish, have dry skin, constipation, or a hoarse voice, or if you've had past thyroid problems or goiter. Signs and symptoms, as well as the results of blood tests that measure thyroid hormone and thyroidstimulating hormone (TSH) levels produced in the pituitary gland, are used to diagnose Hashimoto's disease. A hormone test, done through a blood test, can reveal the amount of hormone produced by the thyroid and pituitary gland. Thyroid hormone levels are low when your thyroid is 
underactive. TSH is raised at the same time because your pituitary gland is attempting to stimulate your thyroid gland to create more thyroid hormone (Malkowski et al., 2019). An antibody test is useful as the creation of aberrant antibodies is the cause of Hashimoto's disease, which is an autoimmune condition. Antibodies against thyroid peroxidase (TPO antibodies), an enzyme ordinarily found in the thyroid gland and involved in the generation of thyroid hormones, can be detected using a blood test. However, not everyone with Hashimoto's thyroiditis has a positive TPO antibody test. Many patients have TPO antibodies but no goiter, hypothyroidism, or other thyroid issues (Malkowski et al., 2019). Previously, doctors couldn't discover an underactive thyroid (hypothyroidism), the primary symptom of Hashimoto's disease, until the disease had progressed. The sensitive TSH test allows doctors to discover thyroid issues early, and in certain cases, before you have any symptoms. Because the TSH test is the most accurate screening tool, your doctor would most likely order a TSH test first and then a thyroid hormone test if necessary. TSH testing are also important in the treatment of hypothyroidism. These tests also assist your doctor in determining the appropriate medicine dosage, both at the outset and over time (Malkowski et al., 2019).

\section{Treatment and management of Hashimoto's disease}

Replacement thyroid hormone is the most common treatment for hypothyroidism. Orally administered titrated levothyroxine sodium is the medicine of choice. It has a 7-day half-life and can be used on a daily basis. To avoid poor absorption, it should not be taken with iron or calcium supplements, aluminum hydroxide, or proton pump inhibitors. For optimal absorption, take it first thing in the morning on an empty stomach (Kawicka et al., 2020). The typical dose is 1.6-1.8 $\mathrm{mcg} / \mathrm{kg}$ per day, but this varies from patient to patient. Patients under the age of 50 should be started on the full dose; however, patients with cardiovascular disorders and the elderly should be given lesser doses. The recommended beginning dose for patients over 50 years old is $25 \mathrm{mcg} / \mathrm{day}$, with reevaluation after six to eight weeks. In contrast, the dose of thyroxine must be increased by $30 \%$ during pregnancy, and larger doses of levothyroxine are required to maintain euthyroid status in patients with short-bowel syndrome (Kawicka et al., 2020). 


\section{Genetic susceptibility}

\section{Human Leukocyte Antigen (HLA) Genes}

The major histocompatibility complex (MHC) region on chromosome 6p21, which encodes human leukocyte antigens (HLAs), was the first gene locus linked to autoimmune thyroid disease. Several immune response genes are found in the HLA area, which is highly polymorphic. The HLA molecule, which is found on antigen presenting cells (APCs), binds and delivers an antigenic peptide, allowing $\mathrm{T}$ cells to recognize and respond to it Chen et al., 2014). HLA alleles with a higher affinity for auto antigenic thyroidal peptides are thought to play a role in the development of autoimmune thyroid disease. However, autoantigen occurrence within the thyroid or thyroid draining lymph nodes is required to induce thyroid autoimmunity, which is followed by HLA presentation. On thyrocytes, abnormal expression of HLA class II molecules has been seen in HT (Eschler et al., 2014).

\section{Cytotoxic T lymphocyte Antigen-4 (CTLA-4) Gene}

On chromosome 2q33, the CTLA-4 gene, which is the second key immune-regulatory gene linked to autoimmune thyroid disease, is located. T-cell activation is suppressed by the production of CTLA-4 on the surface of T cells, which is produced by T-cell receptor activation. CTLA-4 gene polymorphisms may lower CTLA-4 antigen expression or function, resulting in less suppression of T-cell proliferation and, as a result, increased susceptibility to autoimmune responses (Toulis et al., 2010). Several variants of the CTLA-4 gene have been examined in HT patients in the past. Among them, the (AT)n microsatellite CTLA-4 polymorphism in the untranslated region (UTR) was linked to HT in Caucasian and Japanese patients, but not in Italians. A 49A/G single nucleotide polymorphism (SNP) in exon 1 that results in threonine to alanine substitution has been linked to HT, but other investigations have not verified this association. A major meta-analysis of 866 HT patients found a substantial link with 49A/G, using both published and unpublished data (Toulis et al., 2010). 


\section{Thyroglobulin Gene}

Thyroglobulin is a significant thyroid-specific antigen that is also found in the bloodstream, making it an ideal target for the autoimmune response. The thyroglobulin gene is found on chromosome 8q24, and whole genome studies in Japan and the United States were the first to correlate this area to HT and autoimmune thyroid disease. Thyroglobulin gene was discovered to be one of the primary thyroid specific susceptibility genes, related and associated with autoimmune thyroid disease, after precise mapping of this region (Eschler et al., 2014). Later, different alleles of several microsatellite markers and SNPs in the thyroglobulin gene were linked to HT, potentially changing its expression, antigenicity, iodination, or HLA binding. In both Japanese and Caucasian populations, the relationship of the Tgms 2 microsatellite marker in intron 27 with HT was verified. Human thyroglobulin sequencing showed 14 SNPs, four of which were linked to HT, including the exon 10-12 SNP cluster and exon 33 SNP (Chen et al., 2014).

\section{Cytokine Genes and other Immune-Related Genes}

Several genes encoding various inflammatory cytokines have recently been investigated in HT, with some of them impacting the severity of the disease. T-helper type 1 (Th1) cells release interferon (IFN), which increases cell-mediated cytotoxicity, which is the cause of thyroid destruction in HT. The T allele of the +874A/T IFN- SNP, which causes higher IFN- production, has been linked to the severity of hypothyroidism in HT patients (Pearce et al., 2020). Patients with the CC genotype of the -590C/T interleukin 4 (IL-4) SNP had a higher prevalence of severe hypothyroidism, which was linked to a reduced production of IL-4, one of the important Th2 cytokines that suppresses cell-mediated autoimmunity. TGF- gene polymorphism, which inhibits cytokine synthesis, was also linked to HT. The T allele of the $+369 \mathrm{~T} / \mathrm{C}$ SNP, which leads to reduced TGF- secretion, was more common in severe hypothyroidism than in moderate hypothyroidism (Taneri et al., 2019). 


\section{D Model system: spheroids and organoids}

Mallette determined the ability of disrupted and intact cells from adult rat thyroid tissue to reaggregate and reconstruct functional thyroid tissue in 1966 and demonstrated that thyroid cells from trypsinized and intact adult tissue may reform 3D functional thyroid follicles. Despite the fact that thyrocytes were able to reconstruct 3D functional thyroid tissue in vitro as early as the mid-20th century. Antonica in 2012 was able to regenerate thyroid organoids from mouse ESCs (Antonica et al., 2012). The enhanced expression of thyroid specific transcription factors, NKx21 and Pax8, in ESCs leads to the differentiation of thyroid cells that may form 3D and functional follicles in vitro and after transplantation, according to researchers. Kurmann discovered in 2015 that a successful combination of BMP4 and FGF2 signaling caused iPSCs to differentiate into thyroid cells. By isolating thyroid progenitors and culturing them in Matrigel, they were able to create follicular organoids with a single layer of T4/T3 (triiodothyronine) generating thyroid cells. After xenotransplantation, these organoids restored thyroid hormone levels in a mouse model of hypothyroidism (Hollenberg et al., 2017). The lack of animal models for all histopathologic types, especially in rare diseases like anaplastic thyroid cancer, makes organoid technology useful in cancer research. It can also be utilized to construct rare disease models and to look into the future of precision medicine. Furthermore, thyroid organoid model systems might be useful for researching autoimmune thyroid disorders (AITDs), hypothyroidism, hyperthyroidism, cancer, and its genetic and environmental modifiers (Weeber et al., 2017). The long-term goal of regenerative therapy for hypothyroidism is to restore thyroid hormone production in patients. At this time, regenerative medicine based on stem cell technology is a reality, and numerous methods for the creation of differentiated thyroid cells are available. It is now known that endodermal stimulation of Nkx2.1 (NK2 homeobox 1) and Pax8 (paired-box gene 8) promotes the formation of cells capable of producing 3D functional thyroid tissue in vivo (Hollenberg et al., 2017). However, the production of these cells in ESCs or in vivo models is rare, and successful formation of thyroid organoids requires overexpression of Nkx2.1 and Pax8 or target alleles that allow predefined thyroid cells to be sorted and purified. In terms of thyroid regeneration, there is still a long way to go, but recent findings and advancements imply that regenerative therapy for congenital or post-surgical hypothyroidism may be a feasible and achievable objective in the nottoo-distant future (Morshed et al., 2015). Current culture strategies for in vitro 3D models involve 
the use of pluripotent stem cells (PSCs), which can be induced (iPSC), embryonic (ESC) or adult stem cells (ASC). The knowledge gained from several studies in developmental biology has paved the way for the development of methods for the growth of different cell types differentiated from stem cell progenitors.

\section{Spheroid models}

A 3D bioprinter was used to produce thyroid and allantoic spheroids in a collagen hydrogel in 2017. The construct was placed under the renal capsule of hypothyroid mice. After grafting, they noticed vascularization of the construct, as well as restored thyroxine levels and body temperature, indicating functional thyroid regeneration (Bulanova et al., 2017). Bioprinting is the additive biofabrication of three-dimensional (3D) tissues and organ structures using self-assembling tissue spheroids as the building blocks. The thyroid gland is a simple endocrine organ that can be used to test the suggested bioprinting method. A functioning vascularized mouse thyroid gland construct was bioprinted using embryonic tissue spheroids (Hornung et al., 2018). Thyroid tissue was created using the self-assembly principle, using thyroid spheroids (TS) and allantoic spheroids (AS) as sources of thyrocytes and endothelial cells, respectively. Closely placed embryonic tissue spheroids merged into a single integral construct during the culture, EC from AS invaded and vascularized TS, and epithelial cells from the TS gradually produced follicles. Observed the establishment of a capillary network surrounding follicular cells in this experimental scenario, similar to what we see during in utero thyroid development when the thyroid epithelium controls the recruitment, invasion, and extension of EC around follicles (Kohling et al., 2017). Reduced endogenous EC from TS before bioprinting to prove that EC from AS are responsible for vascularization of the thyroid gland construct. Thyroid tissue that had been decreased was totally revascularized with EC from AS. After being grafted under the kidney capsule of hypothyroid mice, the cultured bioprinted construct was able to normalize blood thyroxine levels and body temperature. Bioprinting of a functioning vascularized mouse thyroid gland construct is a step

forward in bioprinting technology, allowing researchers to investigate the self-assembling capabilities of tissue spheroids (Hornung et al., 2017). 


\section{Thyroid organoids}

During embryonic development, the thyroid is formed from the same progenitor cells as the lungs. According to the initial report, temporary overexpression of Pax8 and Nkx2-1 was sufficient to create thyroid organoids from mouse ESCs. Longmire's methodology also produced thyroid organoids by sequentially exposing progenitors to BMP/FGF4, then plating the cells in 3D architecture in Matrigel with thyroid stimulating hormone (TSH) to stimulate thyroid organoids formation (Kurmann et al., 2015). Kurmann et al. (2015) developed a methodology for manufacturing thyroid organoids using mouse PSCs based on this protocol. Thyroid organoids were generated in this fashion, and when transplanted into hypothyroid mice, they secreted thyroid hormones and rescued the mice. The same procedure could be used to produce human thyroid organoids from iPSCs. Kurmann described a method for long term growth of thyroid organoids capable of iodide uptake and thyroglobulin synthesis. Normal thyroid-like tissues emerged after these organoids were implanted into a mouse model of hypothyroidism (Kurmann et al. 2015).

\section{In vitro human thyroid model}

Hundreds of potential TDCs have been identified due to significant advances in the development and implementation of HTS tests for thyroid-related MIEs. An in vitro screening model that more accurately reflects the thyroid gland intrinsic biology could be useful for orthogonal confirmation of tissue level effects on the TH production pathway, lowering the uncertainty associated with HTS experiments (Kosian et al., 2015). The physical and functional properties of 2D and 3D thyroid culture models were characterized using primary human thyrocytes obtained from euthyroid donors. Some features of native thyroid function, such as TSH-dependent gene expression and TG synthesis, were able to be replicated by thyrocytes in a standard 2D monolayer. The $2 \mathrm{D}$ culture format, on the other hand, was definitely lacking in all essential components for thyroid hormonogenesis. In a stable, long-term in vitro culture model, adaptation to a 3D hydrogel culture format using medium optimized for human thyrocytes restored synthesis of iodothyronine hormones for up to 20 days (Degitz et al., 2010). Furthermore, by developing the 3D microtissue model in a 96-well culture format, inhibition of TH production could be reproducibly investigated using a known TDC reference set to directly test the efficacy and potency of TH disruption in a 
concentration-dependent manner. Primary cells, unlike many immortalized cell lines, have stringent criteria for successful cell culture development. The composition of one of the most regularly used primary thyroid cell culture medium formulations was originally designed for FRTL rat thyrocytes (Degitz et al., 2010). Insulin, hydrocortisone, transferrin, glycol-L-histidyl-L-lysine acetate, somatostatin, and TSH are included in the formulation, which is made up of Ham's F-12 base medium supplemented with calf serum and six additives. TSH levels greater than the specified concentration of $0.04 \mathrm{mU} / \mathrm{mL}$ were required for maximum performance, particularly in the 3D culture format, to maximize cellular responses. When cells were maintained at significantly higher levels of $1 \mathrm{mU} / \mathrm{ml} \mathrm{TSH}$, the effects on gene expression, TG protein production, and $\mathrm{TH}$ output were most consistent, hence this concentration in $\mathrm{h} 7 \mathrm{H}$ media was judged best for optimizing the dynamic range in the 3D microtissue test (Kosian et al., 2017).

\section{Impact of Thyroid Autoantibodies Positivity on In Vitro Fertilization}

The female reproductive system's functionality could be significantly impacted by thyroid disease through a variety of methods. Menstrual abnormalities, infertility, anovulation, and poor obstetric outcomes are all linked to overt thyroid illnesses such Hashimoto thyroiditis. Thyroid autoimmunity is the most common pathologic causing thyroid dysfunction in women of reproductive age. When compared to the general population, infertile women have a higher prevalence of thyroid autoimmune dysfunction (Toulis et al., 2010). However, there is a lack of evidence on the direct influence of TAA positivity on IVF success, particularly in the community of infertile, euthyroid TAA positive women. When overt thyroid disease or subclinical hypothyroidism coexist, maternal TAA positive may affect IVF/ICSI results. It's likely that TAA doesn't directly influence oocyte development, embryo implantation, or embryo quality, but rather compromises the physiological processes of implantation through Th1-mediated cytotoxicity (Chen et al., 2014).

\section{Protection against thyroid autoimmunity}

Self-tolerance is maintained through two ways. The thymic elimination of auto reactive $\mathrm{T}$ cells during fetal life is referred to as central tolerance. Those cells that escape central tolerance are prevented from causing autoimmunity by peripheral tolerance mechanisms, in which Tregs play a critical role. They suppress effector T cells, APCs, and B cells, maintaining immunological 
unresponsiveness to self-antigens and reducing an overactive immune response. They can suppress target cells directly or through suppressor cytokines that are produced. Tregs are $\mathrm{T}$ cells that come from the thymus or naive T cells in the periphery, and they express CD25 (the IL-2 receptor chain) and FoxP3. As a result, CD4 CD25 Foxp3 Tregs play a crucial function in the immune system (Pearce et al., 2020). Regulatory suppressive effects are caused by cells with the highest CD25 expression $(\mathrm{CD} 4+\mathrm{CD} 25 \mathrm{high})$. The significance of Tregs in self-tolerance has been widely explored in animal models, particularly in mouse experimental autoimmune thyroiditis, where thymically-derived CD4 CD25 Foxp3 Tregs provided protection against autoimmunity. CD25 expression was higher in HT patients than in healthy persons in humans. The proportion of Tregs was lower intrathyroidal than in peripheral blood in patients with autoimmune thyroid disease (Taneri et al., 2019). Three-color flow cytometry revealed a larger proportion of special Tregs with high levels of FoxP3 in HT patients when compared to controls. This points to a compensatory increase in the Treg subgroup in order to reduce the immunological response. TGF- $\beta$ induces the expression of Foxp3 and the production of Treg cells in Tregs, fibroblasts, macrophages, endothelial cells, and thyrocytes in inflammatory thyroid tissue (Taneri et al., 2019). TGF- $\beta$ promotes suppressive Tregs and inhibits $\mathrm{T}$ cell development, making it a major regulator of immunological tolerance. As a result, serum levels of TGF- $\beta$ were lower in HT patients than in controls, and did not change after therapy with levothyroxine. As a result, TGF- $\beta$ levels appear to be linked to HT rather than thyroid dysfunction. TGF- $\beta$ has been shown to have a suppressive impact in the development of experimental autoimmune thyroiditis. TGF- $\beta$ may trigger the development of fibrosis in the latter stages of thyroiditis (Pearce et al., 2020).

\section{Conclusion}

HT is one of the most common autoimmune disorders, which can be triggered by a variety of factors in genetically predisposed people. Multiple susceptibility genes, some of which are also found in other autoimmune disorders but are unique to thyroid autoimmunity, may be implicated in disease development. Immune-regulatory genes including HLA, CTLA-4, and PTPN22 are now known to play a role in the etiology of HT. Furthermore, recent research has linked cytokine genes like IFN-, IL-4, and TGF- $\beta$ to the development and severity of HT, implying that they influence 
the balance between Th1 and Th2 processes. According to current knowledge, a complicated combination between hereditary and non-genetic variables leads to increased thyroid antigen presentation and decreased immune tolerance, resulting in Th1-type autoimmunity, thyroid destruction, and clinical illness. Despite significant progress in our understanding of HT during the last decade, the exact processes of its growth remain unknown. Hopefully, new information will provide a better understanding of illness causation in the near future, allowing us to detect atrisk individuals and potentially prevent the onset of clinical disease.

\section{References}

Antonica, F., Kasprzyk, D. F., Opitz, R., Iacovino, M., Liao, X. H., Dumitrescu, A. M., \& Costagliola, S. (2012). Generation of functional thyroid from embryonic stem cells. Nature, 491(7422), 66-71.

Aurizio, F., Villalta, D., Metus, P., Doretto, P., \& Tozzoli, R. (2015). Is vitamin D a player or not in the pathophysiology of autoimmune thyroid diseases. Autoimmunity reviews, 14(5), 363-369.

Brown, J., Solomon, D. H., Beall, G. N., Terasaki, P. I., Chopra, I. J., Van Herle, A. J., \& Wu, S. Y. (2012). Autoimmune thyroid diseases-Graves' and Hashimoto's. Annals of Internal Medicine, 88(3), 379-391.

Bulanova, E. A., Koudan, E. V., Degosserie, J., Heymans, C., Pereira, F. D., Parfenov, V. A., \& Mironov, V. A. (2017). Bioprinting of a functional vascularized mouse thyroid gland construct. Biofabrication, 9(3), 034105.

Cappa, M., Bizzarri, C., \& Crea, F. (2011). Autoimmune thyroid diseases in children. Journal of thyroid research, 2011.

Chen, H., Hou, Y., He, J., Cheng, Z., \& Wang, R. (2014). A study on the clinical effect and immunological mechanism in the treatment of Hashimoto's thyroiditis by moxibustion. Journal of traditional Chinese medicine, 13(1), 14-18. 
Degitz, S. J., Korte, L. M., Olson, J. M., Kosian, P. A., Linnum, A. L., \& Tietge, J. E. (2010). Inhibition of thyroid hormone release from cultured amphibian thyroid glands by methimazole, 6-propylthiouracil, and perchlorate. Toxicological Sciences, 118(1), 42-51.

Eschler, D. C., Hasham, A., \& Tomer, Y. (2014). Cutting edge: the etiology of autoimmune thyroid diseases. Clinical reviews in allergy \& immunology, 41(2), 190-197.

Hollenberg, A. N., Choi, J., Serra, M., \& Kotton, D. N. (2017). Regenerative therapy for hypothyroidism: mechanisms and possibilities. Molecular and cellular endocrinology, 445, $35-41$.

Hornung, M. W., Korte, J. J., Olker, J. H., Denny, J. S., Knutsen, C., Hartig, P. C., ... \& Degitz, S. J. (2018). Screening the ToxCast phase 1 chemical library for inhibition of deiodinase type 1 activity. Toxicological Sciences, 162(2), 570-581.

Iddah, M. A., \& Macharia, B. N. (2013). Autoimmune thyroid disorders. International Scholarly Research Notices, 2013.

Kawicka, A., \& Regulska, B. (2020). Metabolic disorders and nutritional status in autoimmune thyroid diseases. Advances in Hygiene \& Experimental Medicine/Postepy Higieny $i$ Medycyny Doswiadczalnej, 69.

Kohling, H. L., Plummer, S. F., Marchesi, J. R., Davidge, K. S., \& Ludgate, M. (2017). The microbiota and autoimmunity: Their role in thyroid autoimmune diseases. Clinical immunology, 183, 63-74.

Kosian, P. A., Haselman, J. T., Korte, J. J., Challis, K., Macherla, C., \& Degitz, S. J. (2015). In vitro, ex vivo, and in vivo determination of thyroid hormone modulating activity of benzothiazoles. Toxicological sciences, 146(2), 254-264.

Kurmann, A. A., Serra, M., Hawkins, F., Rankin, S. A., Mori, M., Astapova, I., \& Kotton, D. N. (2015). Regeneration of thyroid function by transplantation of differentiated pluripotent stem cells. Cell stem cell, 17(5), 527-542.

Morshed, S. A., Latif, R., \& Davies, T. F. (2015). Thyroid cell differentiation from murine induced pluripotent stem cells. Frontiers in endocrinology, 6, 56. 
Malkowski, B., Serafin, Z., Glonek, R., Suwała, S., Lopatto, R., \& Junik, R. (2019). The role of $18 \mathrm{~F}-\mathrm{FDG} \mathrm{PET} / \mathrm{CT}$ in the management of the autoimmune thyroid diseases. Frontiers in endocrinology, 10, 208.

Pastuszak, D., Sewerynek, E., Domanska, D., Gładys, A., Skrzypczak, R., \& Brzezianska, E. (2012). CTLA-4 gene polymorphisms and their influence on predisposition to autoimmune thyroid diseases (Graves' disease and Hashimoto's thyroiditis). Archives of medical science: $A M S, 8(3), 415$.

Pearce, E. N., Farwell, A. P., \& Braverman, L. E. (2020). Thyroiditis. New England Journal of Medicine, 348(26), 2646-2655.

Taneri, F., Yuksel, O., Aydin, A., Tezel, E., \& Onuk, E. (2019). Total thyroidectomy for the treatment of Hashimoto's thyroiditis coexisting with papillary thyroid carcinoma. Advances in therapy, 24(3), 510-516.

Toulis, K. A., Anastasilakis, A. D., Tzellos, T. G., Goulis, D. G., \& Kouvelas, D. (2010). Selenium supplementation in the treatment of Hashimoto's thyroiditis: a systematic review and a meta-analysis. Thyroid, 20(10), 1163-1173.

Weeber, F., Ooft, S. N., Dijkstra, K. K., \& Voest, E. E. (2017). Tumor organoids as a pre-clinical cancer model for drug discovery. Cell Chem Biol. 24: 1092-1100.

Yamada, H., Itoh, M., Hiratsuka, I., \& Hashimoto, S. (2014). Circulating micro RNA s in autoimmune thyroid diseases. Clinical endocrinology, 81(2), 276-281.

\section{Table of Figure}

Adapted from "Thyroid Gland Anatomy and Histology", by BioRender.com (2021). Retrieved from https://app.biorender.com/biorender-templates 